\title{
Segmentation of White Blood Cells Using Fuzzy C Means Segmentation Algorithm
}

\author{
Pronab Kumar Mondal ${ }^{1}$, Uzzal Kumar Prodhan ${ }^{2}$, Md. Selim Al Mamun ${ }^{3}$, \\ Md. Abdur Rahim ${ }^{4}$, Kazi Kamal Hossain ${ }^{5}$ \\ ${ }^{1,2,3}$ Department of CSE, Jatiya Kabi Kazi Nazrul Islam University, Trishal, Mymansingh-2220, Bangladesh. \\ ${ }^{4}$ Department of CSE, Pabna University of Science and Technology, Pabna-6600, Bangladesh. \\ ${ }^{5}$ Southern Institute of Business and Information Technology (SIBIT), Jessore, Bangladesh.
}

\begin{abstract}
The objective of this paper is to develop a tool to identify the white blood cells from blood smear images through segmentation. In our research work, we have identified white blood cells from color images of blood smears. We have used Fuzzy C Means segmentation method and erosion and dilation post processing method to identify the white blood cells. In our obtained result we find that this method successfully identified $98 \%$ of white blood cells from which is a satisfactory result.
\end{abstract}

Keywords: Fuzzy C Mean, Segmentation, Clustering, Cell.

\section{Introduction}

The influence and impact of digital images on modern society is tremendous, and processing of image is now critical component in science and technology. For medical diagnosis, processing of image plays most important role. Image processing is an important phase in order to improve the accuracy both for diagnosis procedure and for surgical operation. The rapid progress in computerized medical image reconstruction, and the associated developments in analysis methods and computer-aided diagnosis, has propelled medical imaging into one of the most important sub-fields in scientific imaging. Identification of white blood cell from blood of smear is very useful for detection of disease in human body. Manually detection of white blood cell is a tiresome, time-consuming and susceptible to error procedure due to the tedious nature of this process $[5,6]$, an automatic system is preferable in this automatic process, identification white blood cell are the most important stages. These reasons have motivated us to select identification of white blood cells. The objective of this paper present to develop an automatic tool to identify the white blood cells from blood of smear. The overview of the work for identifying white blood cell is given below:

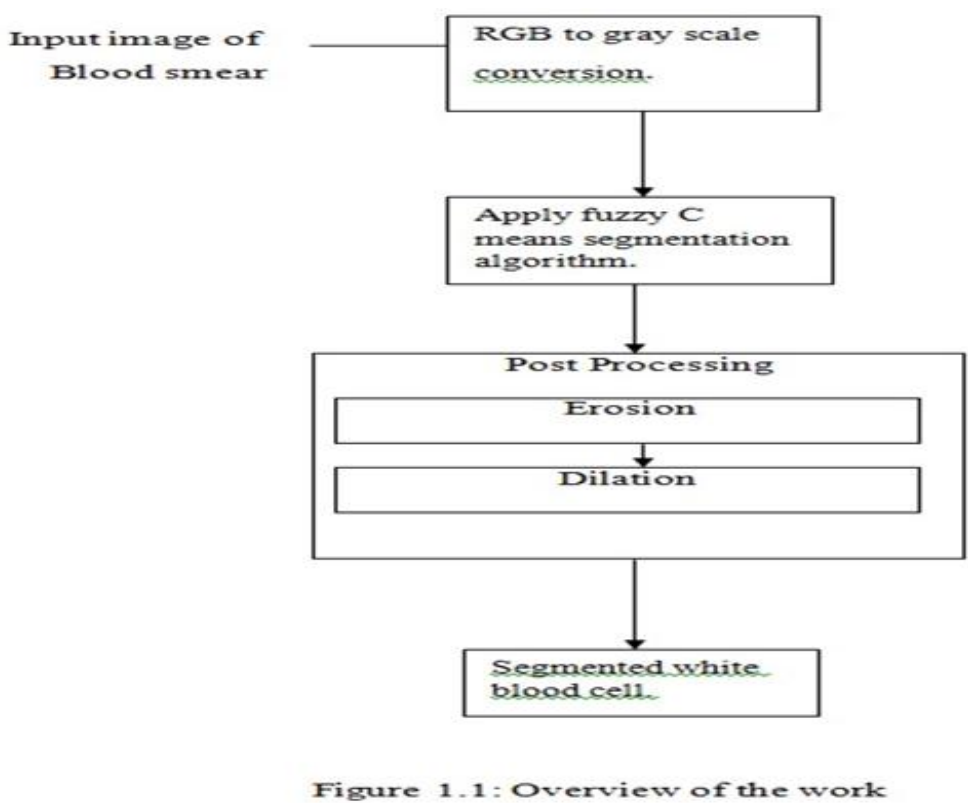




\section{Blood And Blood Cells}

Blood is a specialized bodily fluid that delivers necessary substances to the body's cells (in animals) such as nutrients and oxygen - and transports waste products away from those same cells. In vertebrates, it is composed of blood cells suspended in a liquid called blood plasma. Plasma, which constitutes 55\% of blood fluid, is mostly water ( $92 \%$ by volume),[1] and contains dissipated proteins, glucose, mineral ions, hormones, carbon dioxide (plasma being the main medium for excretory product transportation), platelets and blood cells themselves. The blood cells are mainly red blood cells (also called RBCs or erythrocytes) and white blood cells, including leukocytes and platelets. The most abundant cells in vertebrate blood are red blood cells $[3,4]$. These contain haemoglobin, an iron-containing protein, which facilitates transportation of oxygen by reversibly binding to this respiratory gas and greatly increasing its solubility in blood. In contrast, carbon dioxide is almost entirely transported extracellular dissolved in plasma as bicarbonate ion. Segmentation

There is a large literature on segmentation and clustering, dating back over 30 years, with applications in many areas other than computer vision [2]. In this section we briefly consider some of the related work that is most relevant to our approach: early graph-based methods, region merging techniques, techniques based on mapping image pixels to some feature space and more recent formulations in terms of graph cuts and spectral methods. Finally we briefly consider a class of segmentation methods based on finding minimum cuts in a graph, where the cut criterion is designed in order to minimize the similarity between pixels that are being split. Work by Wu and Leahy [9] introduced such a cut criterion, but it was biased toward finding small components. This bias was addressed with the normalized cut criterion developed by Shi and Malik [10], which takes into account self-similarity of regions. These cut-based approaches to segmentation capture non-local properties of the image, in contrast with the early graph-based methods. However, they provide only a characterization of each cut rather than of the final segmentation. The normalized cut criterion provides a significant advance over the previous work in [9], both from a theoretical and practical point of view (the resulting segmentations capture intuitively salient parts of an image). However, the normalized cut criterion also yields an NP-hard computational problem. While Shi and Malik develop approximation methods for computing the minimum normalized cut, the error in these approximations is not well understood. In practice these approximations are still fairly hard to compute, limiting the method to relatively small images or requiring computation times of several minutes. Recently Weiss [11] has shown how the eigenvector-based approximations developed by Shi and Malik relate to more standard spectral partitioning methods on graphs. However, all such methods are too slow for many practical applications

\section{Fuzzy Logic}

Fuzzy logic is a form of many-valued logic; it deals with reasoning that is fixed or approximate rather than fixed and exact. In contrast with "crisp logic", where binary sets have two-valued logic: true or false, fuzzy logic variables may have a truth value that ranges in degree between 0 and 1. Fuzzy logic has been extended to handle the concept of partial truth, where the truth value may range between completely true and completely false.

\section{Fuzzy C Means Segmentation Technique}

Fuzzy c-means (FCM) is a method of clustering which allows one piece of data to belong to two or more clusters. This method (developed by Dunn in 1973 and improved by Bezdek in 1981) is frequently used in pattern recognition. It is based on minimization of the following objective function:

$$
I_{m}=\sum_{i=1}^{N} \sum_{j=1}^{\odot} u_{i j}^{m}\left\|x_{i}-c_{j}\right\|^{2} \quad, 1 \leq m<\infty
$$

where $\mathrm{m}$ is any real number greater than $1, u_{i j}$ is the degree of membership of $x_{i \bar{i}}$ in the cluster $\mathrm{j}, x_{i \bar{i}}$ is the ith of d-dimensional measured data, $c_{j}$ is the d-dimension center of the cluster, and $\|*\|$ is any norm expressing the similarity between any measured data and the center.

Fuzzy partitioning is carried out through an iterative optimization of the objective function shown above, with the update of membership $u_{i j}$ and the cluster centers $c_{j}$ by:

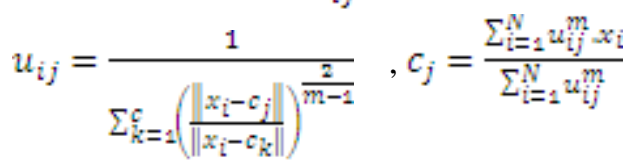


This iteration will stop when $\max _{i j}\left\{\left|u_{i j}^{U^{[k+1)}}-u_{i j}^{U_{j j}^{[k]}}\right|\right\}<\varepsilon \quad$, Where $\varepsilon$ is a termination criterion between 0 and 1 , where as $\mathrm{k}$ are the iteration steps. This procedure converges to a local minimum or a saddle point of $I_{m}$.

The algorithm is composed of the following steps:

1. Initialize $U=\left[u_{i j}\right]$ matrix, $U^{(0)}$

2. At k-step: calculate the centers vectors $c^{(k)}=\left[c_{j}\right]$ with $U^{(k)}$

$$
c_{j}=\frac{\sum_{i=1}^{N} u_{i j}^{m} x_{i}}{\sum_{i=1}^{N} u_{i j}^{m}}
$$

3. Update $U^{(k)}, U^{(k+1)}$

$$
u_{i j}=\frac{1}{\sum_{k=1}^{C}\left(\frac{\left\|x_{i}-c_{j}\right\|}{\| x_{i}-c_{k} \mid}\right)^{\frac{2}{m-1}}}
$$

4. If $\left\|U^{(k+1)}-U^{(k)}\right\|<\varepsilon$ then STOP; Otherwise return to step 2.

\section{Results And Discussion}

In our research we have segmented white blood cell from color images of blood smears. For segmentation we have used Fuzzy C Means segmentation algorithm. The scheme has been applied on 1000 peripheral blood smears images, stained using May-Grunwald-Giesma (MGG) stain [7]. The input images for our research work have been obtained from "Southern Illinonois University" school of medicine's online blood cell sample images, which is provided for research and education. Typical size of the images handled is $256 * 256$.

White blood cells are lager shape and size than any other blood cell. Input images are converted into grayscale images then we perform Fuzzy C Means segmentation algorithm and then post processing technique is used to identify white blood cells from blood smears.

Input blood cells images are

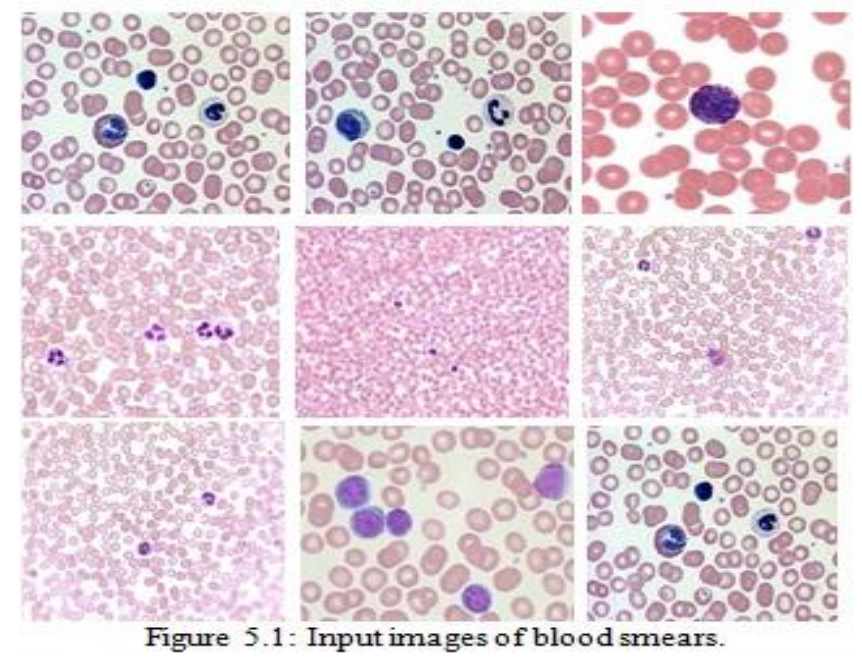

Results after performing Fuzzy C Means segmentation shown in figure 5.2 

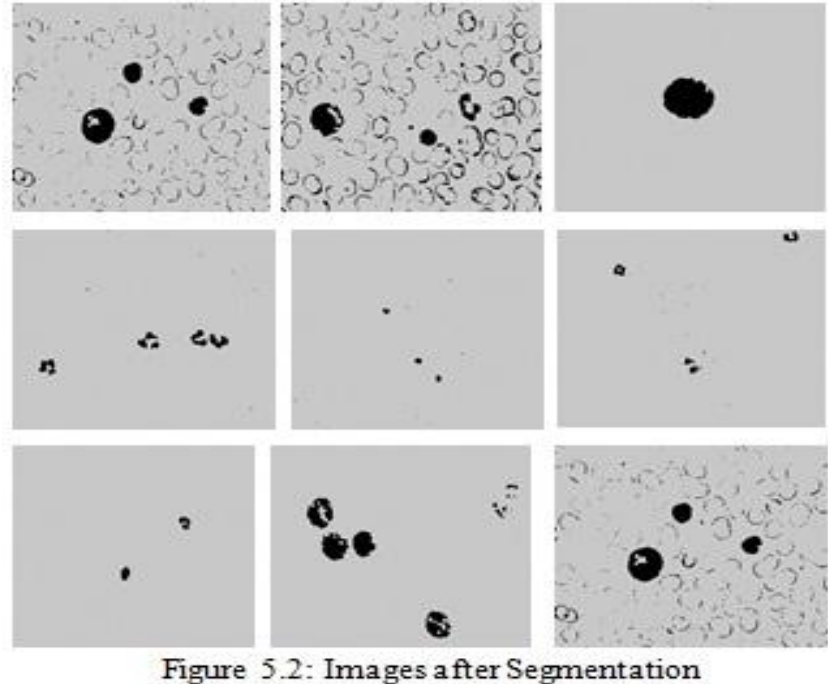

Figure 5.2: Images after Segmentation

Results after post processing segmented image Results after erosion shown in figure 5.3
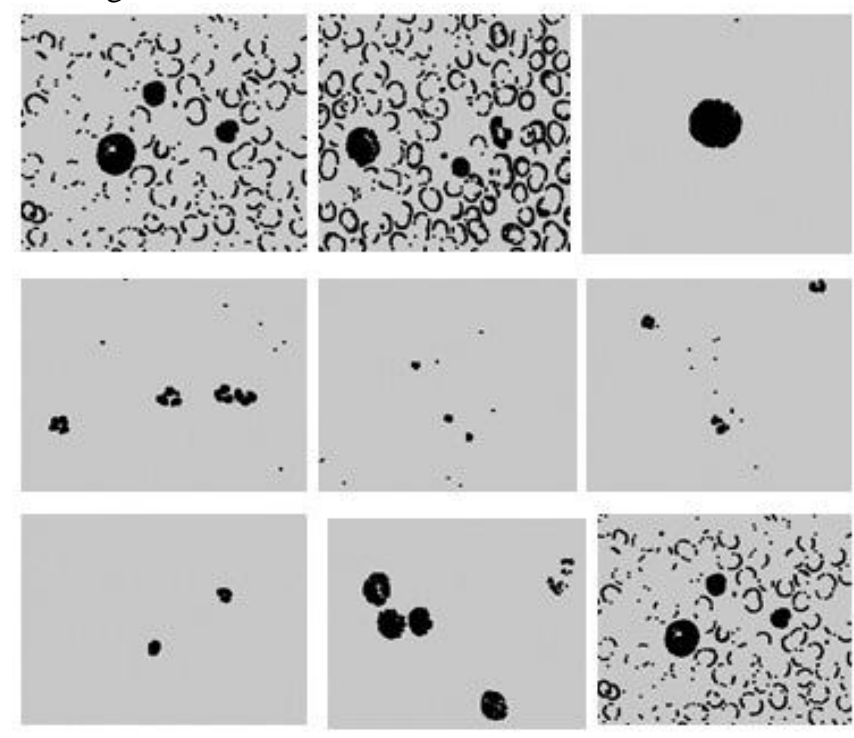

Figure 5.3: Images after erosion.

Results after dilation shown in figure 5.4
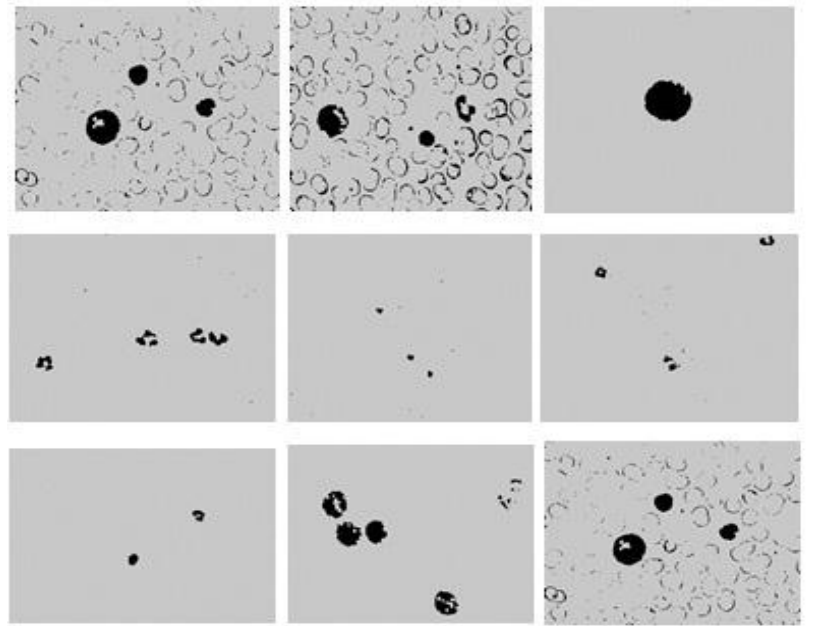

Figure 5.4: Images after dilation 
Table for how many white blood cells identified from our thesis work is shown in table 5.1.

\begin{tabular}{|c|c|c|c|}
\hline Input images & $\begin{array}{c}\text { Actual number of WB } \\
\text { cells }\end{array}$ & $\begin{array}{c}\text { Correctly segmented } \\
\text { blood cells }\end{array}$ & Rate of Percentage (\%) \\
\hline 1000 & 1500 & 1470 & 98 \\
\hline
\end{tabular}

Table 5.1: Predictive accuracy obtained by applying Fuzzy C Means segmentation.

\section{A. Conclusion}

\section{Conclusion And Future Improvement}

In our research, we have identified white blood cells from blood smears. We have used Fuzzy C Means segmentation method and erosion and dilation post processing method to identify the white blood cells. Our method is more reliable and computationally less expensive. In our obtained result we find that this method successfully identified $98 \%$ of white blood cells from which is a satisfactory result.

B. Future Improvement

Future study of our thesis work is counting white cell and blood disorder detection. It is tedious task to locate, identify and count these classes of cells. Due to the importance of these processes, an automated system seems necessary and helpful. White blood cells are clinically more important than red cells and many of blood disorders are related to them. Thus, accurate segmentation of these cells is very important. White blood cell count is used to determine the presence of an infection in the human body.

\section{References}

[1] Cell image segmentation for diagnostic pathology. www.caip.rutgers.edu/riul/research/papers/ps/cell.ps.gz

[2] Single white blood cell extraction in low resolution http://sun12.cecs.missouri.edu/jpark

[3] Cseke. A fast segmentation scheme for white blood cell images. In $11^{\text {th }}$ IAPR Int. conf. of Pattern Recognition, Conf. C: Image, Speech and Signal Analysis, volume 3, pages 530-533, 1992.

[4] D.Wermser, G.Haussman, and C.Liedtke. Segmentation of blood smears by hierarchiclal thresholding. In computer vision, Graphics and Image Processing, volume 25, pages 151-168, 1984.

[5] A.R.Katz. Image analysis and supervised learning in the automated differntiation of white blood cells from microscopic images. Master's thesis, Department of Computer Science, RMIT, Feb.2000.

[6] B.R Kumar, D.k.Joseph, and T.V. Sreenivas. Reager energy based blood cell segmentation. In $14^{\text {th }}$ Intl. Conf. on Digital Signal Processing, pages 925-928, 2002.

[7] N.Otsu. Athreshold selection method from gray level his tograms. IEEE Trans. On System Man and Cyberntics.

[8] Gartner, L. P., \& Hiatt, J. L. (2007). Color Textbook of Histology (3rd ed.). Philadelphia, PA: SAUNDERS Elsevier. pp. 225. ISBN 978-1-4160-2945-8

[9] Nyul, L., Udupa, J., and Zhang, X. (2000). New variants of a method of mri scale standardization. IEEE Transactions on Medical Imaging, 19(2):143-150.

[10] Simmons, A., Tofts, P., Barker, G., and Arridge, S. (1994). Sources of intensity nonuniformity in spin echo images at 1.5 t. Magn Reson Med, 32(1):121-128.

[11] Brown, M. and Semeka, R. (2003). MRI: Basic Principles and Applications. John Wiley and Sons, Inc., 3rd edition 